\title{
Entropies of complex networks with hierarchically constrained topologies
}

\author{
Ginestra Bianconi ${ }^{1}$, Anthony C. C. Coolen ${ }^{2,3}$, Conrad J. Perez Vicente ${ }^{4}$ \\ 1 Abdus Salam International Center for Theoretical Physics, \\ Strada Costiera 11, 34014 Trieste, Italy \\ 2 Department of Mathematics, King's College London, \\ The Strand, London WC2R 2LS, United Kingdom \\ 3 Randall Division of Cell and Molecular Biophysics, \\ Kings College London, New Hunts House, \\ London SE1 1UL, United Kingdom \\ 4 Departament de Física Fonamental, Facultat de Física, \\ Universitat de Barcelona, 08028 Barcelona, Spain
}

\begin{abstract}
The entropy of a hierarchical network topology in an ensemble of sparse random networks, with "hidden variables" associated to its nodes, is the log-likelihood that a given network topology is present in the chosen ensemble. We obtain a general formula for this entropy, which has a clear interpretation in some simple limiting cases. The results provide new keys with which to solve the general problem of "fitting" a given network with an appropriate ensemble of random networks.

PACS numbers: 89.75.Hc, 89.75.Fb, 75.10.Nr
\end{abstract}




\section{INTRODUCTION}

The entropy is a key concept in information theory [1] and in the theory of dynamical systems [2]. In information theory, the problem of inference of a probability distribution on the basis of finite number of independent observations is usually addressed using the maximum likelihood principle or via the minimization of the Kullback-Leibler distance between the given (empirical) distribution and the inferred one. Recently, several studies have extended the tools of information theory along these lines in order to measure the performance of filtering procedures of correlation matrices in the case of multivariate data [3, 4]. In the framework of graph theory the large deviations of the ensemble of random Erdös and Renyi graphs where derived by studying the free energies of statistical mechanics models defined on them [5, 6]. There is now increased interest, in the community of complex networks [7, 8, 9], in the definition of entropy measures that are related with the networks' topological structure [10] or with diffusion processes defined on them [11]. The inference problem applied to complex networks can be formulated as the identification of the ensemble of networks which retains the essential structural characteristics and complexity of a given real network realization. The identification of this ensemble, is an active field of research. One aims to fit a given specific network with a suitable network ensemble that retains some information on its structure. Newman has proposed this approach to find the community structure in a given network [12]. Later, this method has been extended to define ensembles of networks that have other topological characteristics in common with the real network, such as the degree sequence and/or the degree correlations. As we add further features that a desired ensemble is to have in common with a given real network, we effectively consider ensembles with decreasing cardinality. The cardinality of an ensemble of networks with a given topology has attracted the attention of the graph theory community [13, 14, 15], and more recently also of the statistical mechanics community [10].

In this paper we evaluate the entropy of a given hierarchical topology in a "canonical" or "hidden variable" ensemble, i.e. we calculate the normalized logarithm of the probability that a given topology appears in this ensemble. By hierarchical topology we will mean the set of the generalized degrees of the nodes, defined as the sequence $\boldsymbol{k}_{i}=\left(k_{i}^{1}, k_{i}^{2}, \ldots, k_{i}^{L}\right)$ of the number of nodes at distance $1,2, \ldots, L$ from the node $i$. The "canonical" or "hidden variable" [16, 17, 18, 19, 20] ensembles are generalization of the $G(N, p)$ ensemble for het- 
erogeneous nodes. The hetereogeneity of the nodes is described in terms of some "hidden variables" $x_{i}$, defined on each node $i$ of the network, and the probability $p_{i j}$ of a link between a node $i$ and a node $j$ is not $p$ as in $G(N, p)$ but it is a general function $Q\left(x_{i}, x_{j}\right)$ of the hidden variables at $i$ and $j$ nodes. These ensembles correspond to networks which satisfy soft constraints, for example the degree of a node is not fixed, but only the average degree of each node is fixed, allowing for Poissonian fluctuations.

We derive a general formula for the entropy of a given topology in a "canonical" ensemble using ideas and methods from the study of diluted combinatorial optimization problems and statistical mechanical systems on sparse networks [21, 22, 23, 24, 25, 26, 27, 28, 29, 30, 31, 32, 33, 34, 35, 36, 37, 38, 39, 40, 41]. In the simple case where we study the likelihood of a degree distribution of a network belonging to the chosen ensemble the entropy is found to be the Kullback-Leibler distance between the probability distribution of the degrees and the expected probability of the typical topology of the network.

The paper is structured as follows: in section II we introduce the definition of the problem, in section III we provide the asymptotic entropy expression of the network topology in a given ensemble, in section IV we study the form that the entropy takes in special and relevant cases, and the conclusions are presented in section $\mathrm{V}$.

\section{FORMULATION OF THE PROBLEM AND DEFINITIONS}

To model the essential properties of a real network it is useful to think of it as an instance of an ensemble of networks. The ensemble can be either "microcanonical" or "canonical" depending on whether the networks in the ensemble are subject to hard or soft constraints. The main example of what we call a "microcanonical" ensemble is $G(N, M)$ where the number of links is fixed to be exactly $M$, and the main example of "canonical" ensemble is $G(N, p)$ in which only the average number of links $\langle M\rangle=p N(N-1) / 2$ is fixed. These ensembles can be generalized to ensembles of random graphs with a given degree sequence and with a given hidden variable distribution. In this paper we will calculate the entropy of a given network topology (defined in terms of its hierarchical structure) in a general "canonical" ensemble. This entropy is defined as the probability that the given network topology is found in the "canonical" network ensemble under consideration. 


\section{A. "Canonical" ensembles}

We consider networks characterized by $N$ nodes (or 'sites') labeled $i=1, \ldots, N$, and a symmetric matrix $\boldsymbol{c}$ with entries $c_{i j} \in\{0,1\}$ that specify whether $\left(c_{i j}=1\right)$ or not $\left(c_{i j}=\right.$ 0 ) nodes $i$ and $j$ are connected. We choose $c_{i i}=0$ for all $i$. We write the set of all

such undirected networks as $\mathcal{G}=\{0,1\}^{\frac{1}{2} N(N-1)}$. On this set $\mathcal{G}$ we introduce the following probability measure, in order to define an ensemble $\{\mathcal{G}, W\}$ of random networks:

$$
W(\boldsymbol{c} \mid \boldsymbol{x})=\prod_{i<j}\left[\frac{c}{N} Q\left(x_{i}, x_{j}\right) \delta_{c_{i j}, 1}+\left(1-\frac{c}{N} Q\left(x_{i}, x_{j}\right)\right) \delta_{c_{i j}, 0}\right]
$$

The $\left\{x_{i}\right\}$ represent 'hidden variables', drawn for each site independently with statistics $p(x)$ to be defined later, and the function $Q\left(x, x^{\prime}\right) \geq 0$ is chosen such that $\sum_{x x^{\prime}} p(x) p\left(x^{\prime}\right) Q\left(x, x^{\prime}\right)=1$. The latter condition ensures that asymptotically $c$ represents the average connectivity, viz. $\lim _{N \rightarrow \infty}\left\langle N^{-1} \sum_{i j} c_{i j}\right\rangle=c$. Note that throughout this paper the 'hidden variables' $\left\{x_{i}\right\}$ can be scalar, discrete or multidimensional.

\section{B. Hierarchical constraints topologies}

Next we introduce a hierarchy of single-site observables with the objective to characterize with increasing precision the local topology of a network $\boldsymbol{c} \in \mathcal{G}$. They can be interpreted as generalized degrees $\boldsymbol{k}_{i}(\boldsymbol{c})=\left(k_{i}^{1}(\boldsymbol{c}), \ldots, k_{i}^{L}(\boldsymbol{c})\right)$ of individual nodes $i$ :

$$
k_{i}^{\ell}(\boldsymbol{c})=\sum_{j_{1} \ldots j_{\ell}} c_{i j_{1}} c_{j_{1} j_{2}} \ldots c_{j_{\ell-1} j_{\ell}} \in\left\{0,1,2, \ldots, N^{\ell}\right\}
$$

In the absence of local loops, $k_{i}^{\ell}(\boldsymbol{c})$ measures the size (measured in number of nodes) of the local environment of node $i$, at a distance of $\ell$ links. However, in this tree the nodes are counted with a multiplicity equal to their number of descendants encountered; similarly, in the case of local loops, nodes that can be visited from site $i$ via multiple routes of length $\leq \ell$ are counted with this multiplicity. Note that $k_{i}^{1}(\boldsymbol{c})=\sum_{j} c_{i j}$ is the ordinary degree of node $i$, and that (2) can also be written as

$$
k_{i}^{1}(\boldsymbol{c})=\sum_{j} c_{i j}, \quad k_{i}^{\ell+1}(\boldsymbol{c})=\sum_{j} c_{i j} k_{j}^{\ell}(\boldsymbol{c})
$$

By definition, if $k_{i}^{1}(\boldsymbol{c})=0$ then $k_{i}^{\ell}(\boldsymbol{c})=0$ for all $\ell$. It is now natural to characterize the global topology of a network $\boldsymbol{c}$ either by giving its $N$ generalized degree vectors $\left\{\boldsymbol{k}_{1}(\boldsymbol{c}), \ldots, \boldsymbol{k}_{N}(\boldsymbol{c})\right\}$ 
themselves, or by giving the collective generalized degree statistics, conditioned on the values of the hidden variables, i.e.

$$
P(\boldsymbol{k} \mid x, \boldsymbol{c})=P\left(k_{1}, \ldots, k_{L} \mid x, \boldsymbol{c}\right)=\frac{1}{N p(x)} \sum_{i=1}^{N} \delta_{\boldsymbol{k}, \boldsymbol{k}_{i}(\boldsymbol{c})} \delta\left(x-x_{i}\right)
$$

We adopt the convention that always $\boldsymbol{k}=\left(k_{1}, \ldots, k_{L}\right) \in \mathbb{N}^{L}$, unless indicated otherwise.

\section{Entropy of a network contraint topology in a given ensemble}

Our goal is to quantify to what extent the above characterization of networks, by the generalized degrees $\{\boldsymbol{k}\} \equiv\left\{\boldsymbol{k}_{1}, \ldots, \boldsymbol{k}_{N}\right\}$ or by the degree statistics $P_{L}(\boldsymbol{k})$, specifies their micro-structure. This can be measured by the effective number of networks in the ensemble $\{\mathcal{G}, W\}$ that meet the relevant contraints, i.e. (apart from a constant) by the Boltzmann entropies:

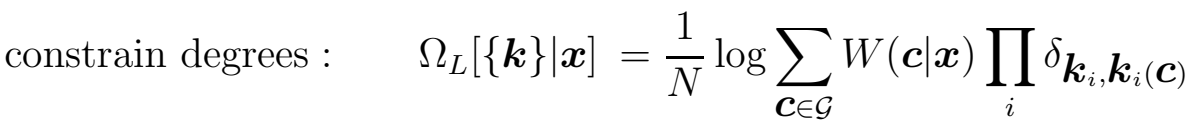

constrain statistics : $\quad \Omega_{L}[P \mid \boldsymbol{x}]=\frac{1}{N} \log \sum_{\boldsymbol{c} \in \mathcal{G}} W(\boldsymbol{c} \mid \boldsymbol{x}) \prod_{\boldsymbol{k}, x} \delta[P(\boldsymbol{k} \mid x)-P(\boldsymbol{k} \mid x, \boldsymbol{c})]$

$$
=\frac{1}{N} \log \sum_{\boldsymbol{k}_{1} \ldots \boldsymbol{k}_{N}} \prod_{\boldsymbol{k}_{, x}} \delta\left[P(\boldsymbol{k} \mid x)-\frac{\sum_{i} \delta_{\boldsymbol{k}, \boldsymbol{k}_{i}} \delta\left[x-x_{i}\right]}{N p(x)}\right] e^{N \Omega_{L}[\{\boldsymbol{k}\} \mid \boldsymbol{x}]}
$$

The larger $\Omega_{L}[\ldots]$, the larger the effective number of graphs with the imposed global topology, viz. $\left\{\boldsymbol{k}_{i}\right\}$ or $P(\boldsymbol{k} \mid x)$, so the less specific is the proposed macroscopic topology characterization. We will find that generally $\Omega_{L}[\ldots]=\mathcal{O}\left(N^{0}\right)$ as $N \rightarrow \infty$. The remainder of this paper deals with the calculation of (51) and (6) in the limit $N \rightarrow \infty$, and their dependence on the choices made for $P(\boldsymbol{k} \mid x)$ and the for ensemble characteristics as defined by $p(x)$ and $Q\left(x, x^{\prime}\right)$

\section{ASYMPTOTIC VALUES OF THE ENTROPY OF NETWORK TOPOLOGY IN A GIVEN ENSEMBLE}

\section{A. Derivation of steepest descent extremization formulas}

Since the ensemble (11) is invariant under all node permutations, the difference between the two formulae (5,6) should reflect only the node permutation freedom that is present in 
(6) but absent from (5). We evaluate (56) by writing each Kronecker $\delta$ and each $\delta$-function in integral form. Upon defining the short-hands $k_{i}^{0}=1$ for all $i$, and $\boldsymbol{\omega}_{i} \cdot \boldsymbol{k}_{i}=\sum_{\ell=1}^{L} \omega_{i}^{\ell} k_{i}^{\ell}$, expression (3) allows us to simplify the term $\delta_{\boldsymbol{k}, \boldsymbol{k}_{i}(\boldsymbol{c})}$ to

$$
\delta_{\boldsymbol{k}_{i}, \boldsymbol{k}_{i}(\boldsymbol{c})}=\int_{-\pi}^{\pi} \frac{d \boldsymbol{\omega}_{i} e^{i \boldsymbol{\omega}_{i} \cdot \boldsymbol{k}_{i}}}{(2 \pi)^{L}} e^{-i \sum_{j} c_{i j} \sum_{\ell=1}^{L} \omega_{i}^{\ell} k_{j}^{\ell-1}}
$$

We next define

$$
D[\{\boldsymbol{\omega}, \boldsymbol{k}\} \mid \boldsymbol{x}]=\sum_{\boldsymbol{c} \in \mathcal{G}} W(\boldsymbol{c} \mid \boldsymbol{x}) e^{-i \sum_{i j} c_{i j} \sum_{\ell=1}^{L} \omega_{i}^{\ell} k_{j}^{\ell-1}}
$$

and subsequently find our that our two entropies can be written in the form

$$
\begin{aligned}
\Omega_{L}[\{\boldsymbol{k}\} \mid \boldsymbol{x}]= & \frac{1}{N} \log \int_{-\pi}^{\pi} \prod_{i}\left[\frac{d \boldsymbol{\omega}_{i} e^{i \boldsymbol{\omega}_{i} \cdot \boldsymbol{k}_{i}}}{(2 \pi)^{L}}\right] D[\{\boldsymbol{\omega}, \boldsymbol{k}\} \mid \boldsymbol{x}] \\
\Omega_{L}[P \mid \boldsymbol{x}]= & \frac{1}{N} \log \int \prod_{\boldsymbol{k}_{x}}\left[\frac{d \hat{P}(\boldsymbol{k} \mid x) e^{i N \hat{P}(\boldsymbol{k} \mid x) P(\boldsymbol{k} \mid x)}}{2 \pi / N}\right] \sum_{\boldsymbol{k}_{1} \ldots \boldsymbol{k}_{N}} e^{N \Omega_{L}[\{\boldsymbol{k}\} \mid \boldsymbol{x}]-i \sum_{i} \hat{P}\left(\boldsymbol{k}_{i} \mid x_{i}\right)} \\
= & \frac{1}{N} \lim _{\Delta \rightarrow 0} \log \int \prod_{\boldsymbol{k}_{, x}}\left[\frac{d \hat{P}(\boldsymbol{k} \mid x) e^{i N \Delta \hat{P}(\boldsymbol{k} \mid x) P(\boldsymbol{k} \mid x)}}{2 \pi / N \Delta}\right] \\
& \times \sum_{\boldsymbol{k}_{1} \ldots \boldsymbol{k}_{N}} \int_{-\pi}^{\pi} \prod_{i}\left[\frac{d \boldsymbol{\omega}_{i} e^{i\left[\boldsymbol{\omega}_{i} \cdot \boldsymbol{k}_{i}-\hat{P}\left(\boldsymbol{k}_{i} \mid x_{i}\right) / p\left(x_{i}\right)\right]}}{(2 \pi)^{L}}\right] D[\{\boldsymbol{\omega}, \boldsymbol{k}\} \mid \boldsymbol{x}]
\end{aligned}
$$

The core of the problem is apparently to calculate the function $D[\{\boldsymbol{\omega}, \boldsymbol{k}\}]$ in (8)), which involves the introduction of a measure $W(\boldsymbol{\omega}, \boldsymbol{k}, x \mid\{\boldsymbol{\omega}, \boldsymbol{k}\})=N^{-1} \sum_{i} \delta_{\boldsymbol{k}, \boldsymbol{k}_{i}} \delta\left[x-x_{i}\right] \delta\left[\boldsymbol{\omega}-\boldsymbol{\omega}_{i}\right]$ :

$$
\begin{aligned}
D[\{\boldsymbol{\omega}, \boldsymbol{k}\} \mid \boldsymbol{x}]= & \prod_{i<j}\left\{1+\frac{c}{N} Q\left(x_{i}, x_{j}\right)\left[e^{-i \sum_{\ell=1}^{L}\left[\omega_{i}^{\ell} k_{j}^{\ell-1}+\omega_{j}^{\ell} k_{i}^{\ell-1}\right]}-1\right]\right\} \\
= & \exp \left\{\frac{1}{2} c N \int d x d x^{\prime} Q\left(x, x^{\prime}\right) \int_{-\pi}^{\pi} d \boldsymbol{\omega} d \boldsymbol{\omega}^{\prime} \sum_{\boldsymbol{k} \boldsymbol{k}^{\prime}} W(\boldsymbol{\omega}, \boldsymbol{k}, x \mid \ldots)\right. \\
& \left.\times W\left(\boldsymbol{\omega}^{\prime}, \boldsymbol{k}^{\prime}, x^{\prime} \mid \ldots\right)\left[e^{-i \sum_{\ell=1}^{L}\left[\omega_{\ell} k_{\ell-1}^{\prime}+\omega_{\ell}^{\prime} k_{\ell-1}\right]}-1\right]+\mathcal{O}\left(N^{0}\right)\right\}
\end{aligned}
$$

We isolate $W(\ldots \mid \ldots)$ via suitable integrations over $\delta$-functions, using the functional measure $\{d W\}=\lim _{\Delta \boldsymbol{\omega} \rightarrow \mathbf{0}} \lim _{\Delta x \rightarrow 0} \prod_{\boldsymbol{\omega}, \boldsymbol{k}, x}[d W(\boldsymbol{\omega}, \boldsymbol{k}, x) \Delta \boldsymbol{\omega} \Delta x \sqrt{N / 2 \pi}]$, resulting in

$$
\begin{aligned}
D[\{\boldsymbol{\omega}, \boldsymbol{k}\}]= & \int\{d W d \hat{W}\} e^{i N \int_{-\pi}^{\pi} d \boldsymbol{\omega} d x \sum_{\boldsymbol{k}} \hat{W}(\boldsymbol{\omega}, \boldsymbol{k}, x) W(\boldsymbol{\omega}, \boldsymbol{k}, x)+\mathcal{O}\left(N^{0}\right)} \\
& \times e^{\frac{1}{2} c N \int d x d x^{\prime} Q\left(x, x^{\prime}\right) \int_{-\pi}^{\pi} d \boldsymbol{\omega} d \boldsymbol{\omega}^{\prime} \sum_{\boldsymbol{k}} \boldsymbol{k}^{\prime} W(\boldsymbol{\omega}, \boldsymbol{k}, x) W\left(\boldsymbol{\omega}^{\prime}, \boldsymbol{k}^{\prime}, x^{\prime}\right)\left[e^{-i \sum_{\ell=1}^{L}\left[\omega_{\ell} k_{\ell-1}^{\prime}+\omega_{\ell}^{\prime} k_{\ell-1}\right]}-1\right]} \\
& \times e^{-i \sum_{i} \hat{W}\left(\boldsymbol{\omega}_{i}, \boldsymbol{k}_{i}, x_{i}\right)}
\end{aligned}
$$


Now only the last line contains microscopic variables, and it factorizes fully over the nodes of the network. Upon inserting (12) into (9) and (10) this allows us to evaluate both expressions for $N \rightarrow \infty$ via steepest descent integration over the distributions $W(\boldsymbol{\omega}, \boldsymbol{k}, x)$, leading to

$$
\begin{aligned}
\Omega_{L}[\{\boldsymbol{k}\} \mid \boldsymbol{x}] & =\operatorname{extr}_{\{W, \hat{W}\}} \Psi_{1}[\{W, \hat{W}\}] \\
\Omega_{L}[P \mid \boldsymbol{x}] & =\operatorname{extr}_{\{W, \hat{W}, \hat{P}\}} \Psi_{2}[\{W, \hat{W}, \hat{P}\}]
\end{aligned}
$$

with the functions

$$
\begin{aligned}
\Psi_{1}[\{W, \hat{W}\}]= & i \int_{-\pi}^{\pi} d \boldsymbol{\omega} d x \sum_{\boldsymbol{k}} \hat{W}(\boldsymbol{\omega}, \boldsymbol{k}, x) W(\boldsymbol{\omega}, \boldsymbol{k}, x)+\Phi[\{W\}] \\
& +\int d x p(x) \sum_{\boldsymbol{k}} P(\boldsymbol{k} \mid x) \log \int_{-\pi}^{\pi} \frac{d \boldsymbol{\omega}}{(2 \pi)^{L}} e^{i[\boldsymbol{\omega} \cdot \boldsymbol{k}-\hat{W}(\boldsymbol{\omega}, \boldsymbol{k}, x)]} \\
\Psi_{2}[\{W, \hat{W}, \hat{P}\}]= & i \int_{-\pi}^{\pi} d \boldsymbol{\omega} d x \sum_{\boldsymbol{k}} \hat{W}(\boldsymbol{\omega}, \boldsymbol{k}, x) W(\boldsymbol{\omega}, \boldsymbol{k}, x)+\Phi[\{W\}] \\
& +i \int d x \sum_{\boldsymbol{k}} \hat{P}(\boldsymbol{k} \mid x) P(\boldsymbol{k} \mid x) \\
& +\int d x p(x) \log \int_{-\pi}^{\pi} \frac{d \boldsymbol{\omega}}{(2 \pi)^{L}} \sum_{\boldsymbol{k}} e^{i[\boldsymbol{\omega} \cdot \boldsymbol{k}-\hat{P}(\boldsymbol{k} \mid x) / p(x)-\hat{W}(\boldsymbol{\omega}, \boldsymbol{k}, x)]}
\end{aligned}
$$

where

$$
\begin{gathered}
\Phi[\{W\}]=\frac{1}{2} c \int d x d x^{\prime} Q\left(x, x^{\prime}\right) \int_{-\pi}^{\pi} d \boldsymbol{\omega} d \boldsymbol{\omega}^{\prime} \sum_{\boldsymbol{k}^{\prime}} W(\boldsymbol{\omega}, \boldsymbol{k}, x) W\left(\boldsymbol{\omega}^{\prime}, \boldsymbol{k}^{\prime}, x^{\prime}\right) \\
\times\left[e^{-i \sum_{\ell=1}^{L}\left[\omega_{\ell} k_{\ell-1}^{\prime}+\omega_{\ell}^{\prime} k_{\ell-1}\right]}-1\right]
\end{gathered}
$$

It will be convenient to introduce new functions $Q(\boldsymbol{k} \mid x)=\exp [-i \hat{P}(\boldsymbol{k} \mid x) / p(x)]$ and $V(\boldsymbol{\omega}, \boldsymbol{k}, x)=\exp [-i \hat{W}(\boldsymbol{\omega}, \boldsymbol{k}, x)]$ so that our saddle-point equations simplify to

$$
\begin{aligned}
\Omega_{L}[\{\boldsymbol{k}\} \mid \boldsymbol{x}] & =\operatorname{extr}_{\{V, W\}} \tilde{\Psi}_{1}[\{V, W\}] \\
\Omega_{L}[P \mid \boldsymbol{x}] & =\operatorname{extr}_{\{Q, V, W\}} \tilde{\Psi}_{2}[\{Q, V, W\}]
\end{aligned}
$$

with the functions

$$
\begin{aligned}
\tilde{\Psi}_{1}[\{V, W\}]= & \Phi[\{W\}]-\int_{-\pi}^{\pi} d \boldsymbol{\omega} d x \sum_{\boldsymbol{k}} W(\boldsymbol{\omega}, \boldsymbol{k}, x) \log V(\boldsymbol{\omega}, \boldsymbol{k}, x) \\
& +\int d x p(x) \sum_{\boldsymbol{k}} P(\boldsymbol{k} \mid x) \log \int_{-\pi}^{\pi} \frac{d \boldsymbol{\omega}}{(2 \pi)^{L}} V(\boldsymbol{\omega}, \boldsymbol{k}, x) e^{i \boldsymbol{\omega} \cdot \boldsymbol{k}}
\end{aligned}
$$




$$
\begin{aligned}
\tilde{\Psi}_{2}[\{Q, V, W\}]= & \Phi[\{W\}]-\int_{-\pi}^{\pi} d \boldsymbol{\omega} d x \sum_{\boldsymbol{k}} W(\boldsymbol{\omega}, \boldsymbol{k}, x) \log V(\boldsymbol{\omega}, \boldsymbol{k}, x) \\
& -\int d x p(x) \sum_{\boldsymbol{k}} P(\boldsymbol{k} \mid x) \log Q(\boldsymbol{k} \mid x) \\
& +\int d x p(x) \log \sum_{\boldsymbol{k}} Q(\boldsymbol{k} \mid x) \int_{-\pi}^{\pi} \frac{d \boldsymbol{\omega}}{(2 \pi)^{L}} V(\boldsymbol{\omega}, \boldsymbol{k}, x) e^{i \boldsymbol{\omega} \cdot \boldsymbol{k}}
\end{aligned}
$$

\section{B. Simplification and reduction of the functional saddle-point equations}

We can now do the functional variations of $\tilde{\Psi}_{1}[\ldots]$ and $\tilde{\Psi}_{2}[\ldots]$ and find our saddlepoint equations from which to solve $\{Q, V, W\}$. For $\tilde{\Psi}_{1}[\ldots]$ (referring to ensembles with constrained generalized degrees) these are found to be the following:

$$
\begin{aligned}
\log V(\boldsymbol{\omega}, \boldsymbol{k}, x) & =c \int d x^{\prime} Q\left(x, x^{\prime}\right) \int_{-\pi}^{\pi} d \boldsymbol{\omega}^{\prime} \sum_{\boldsymbol{k}^{\prime}} W\left(\boldsymbol{\omega}^{\prime}, \boldsymbol{k}^{\prime}, x^{\prime}\right)\left[e^{-i \sum_{\ell=1}^{L}\left[\omega_{\ell} k_{\ell-1}^{\prime}+\omega_{\ell}^{\prime} k_{\ell-1}\right]}-1\right] \\
W(\boldsymbol{\omega}, \boldsymbol{k}, x) & =\frac{p(x) P(\boldsymbol{k} \mid x) V(\boldsymbol{\omega}, \boldsymbol{k}, x) e^{i \boldsymbol{\omega} \cdot \boldsymbol{k}}}{\int_{-\pi}^{\pi} d \boldsymbol{\omega}^{\prime} V\left(\boldsymbol{\omega}^{\prime}, \boldsymbol{k}, x\right) e^{i \boldsymbol{\omega}^{\prime} \cdot \boldsymbol{k}}}
\end{aligned}
$$

For $\tilde{\Psi}_{2}[\ldots]$ (referring to ensembles with constrained distributions of generalized degrees) these are found to be the following:

$$
\begin{aligned}
\log V(\boldsymbol{\omega}, \boldsymbol{k}, x) & =c \int d x^{\prime} Q\left(x, x^{\prime}\right) \int_{-\pi}^{\pi} d \boldsymbol{\omega}^{\prime} \sum_{\boldsymbol{k}^{\prime}} W\left(\boldsymbol{\omega}^{\prime}, \boldsymbol{k}^{\prime}, x^{\prime}\right)\left[e^{-i \sum_{\ell=1}^{L}\left[\omega_{\ell} k_{\ell-1}^{\prime}+\omega_{\ell}^{\prime} k_{\ell-1}\right]}-1\right] \\
W(\boldsymbol{\omega}, \boldsymbol{k}, x) & =\frac{p(x) Q(\boldsymbol{k} \mid x) V(\boldsymbol{\omega}, \boldsymbol{k}, x) e^{i \boldsymbol{\omega} \cdot \boldsymbol{k}}}{\sum_{\boldsymbol{k}^{\prime}} Q\left(\boldsymbol{k}^{\prime} \mid x\right) \int_{-\pi}^{\pi} d \boldsymbol{\omega}^{\prime} V\left(\boldsymbol{\omega}^{\prime}, \boldsymbol{k}^{\prime}, x\right) e^{i \boldsymbol{\omega}^{\prime} \cdot \boldsymbol{k}^{\prime}}} \\
P(\boldsymbol{k} \mid x) & =\frac{Q(\boldsymbol{k} \mid x) \int_{-\pi}^{\pi} d \boldsymbol{\omega} V(\boldsymbol{\omega}, \boldsymbol{k}, x) e^{i \boldsymbol{\omega} \cdot \boldsymbol{k}}}{\sum_{\boldsymbol{k}^{\prime}} Q\left(\boldsymbol{k}^{\prime} \mid x\right) \int_{-\pi}^{\pi} d \boldsymbol{\omega} V\left(\boldsymbol{\omega}, \boldsymbol{k}^{\prime}, x\right) e^{i \boldsymbol{\omega} \cdot \boldsymbol{k}^{\prime}}}
\end{aligned}
$$

The last equation is easily solved, viz.

$$
Q(\boldsymbol{k} \mid x)=\frac{P(\boldsymbol{k} \mid x)}{\int_{-\pi}^{\pi} d \boldsymbol{\omega} V(\boldsymbol{\omega}, \boldsymbol{k}, x) e^{i \boldsymbol{\omega} \cdot \boldsymbol{k}}}
$$

whereas in both cases (constrained degrees versus constrained degree statistics) we can eliminate immediately the kernels $W(\boldsymbol{\omega}, \boldsymbol{k}, x)$, leaving us in either case with a closed problem for the kernel $V(\boldsymbol{\omega}, \boldsymbol{k}, x)$ only. Upon inserting the solution (27) into (26) one finds that this remaining problem is in fact identical for both types of constraints, namely

$$
\log V(\boldsymbol{\omega}, \boldsymbol{k}, x)=c \int d x^{\prime} p\left(x^{\prime}\right) Q\left(x, x^{\prime}\right) \sum_{\boldsymbol{k}^{\prime}} P\left(\boldsymbol{k}^{\prime} \mid x^{\prime}\right)
$$




$$
\times \frac{\int_{-\pi}^{\pi} d \boldsymbol{\omega}^{\prime} V\left(\boldsymbol{\omega}^{\prime}, \boldsymbol{k}^{\prime}, x^{\prime}\right) e^{i \boldsymbol{\omega}^{\prime} \cdot \boldsymbol{k}^{\prime}}\left[e^{-i \sum_{\ell=1}^{L}\left[\omega_{\ell} k_{\ell-1}^{\prime}+\omega_{\ell}^{\prime} k_{\ell-1}\right]}-1\right]}{\int_{-\pi}^{\pi} d \boldsymbol{\omega}^{\prime} V\left(\boldsymbol{\omega}^{\prime}, \boldsymbol{k}^{\prime}, x^{\prime}\right) e^{i \boldsymbol{\omega}^{\prime} \cdot \boldsymbol{k}^{\prime}}}
$$

In addition one finds that (23) holds in both cases. The solution of (28) is of the form

$$
V(\boldsymbol{\omega}, \boldsymbol{k}, x)=e^{-c \int d x^{\prime} Q\left(x, x^{\prime}\right) p\left(x^{\prime}\right)} \exp \left[c \sum_{\boldsymbol{\xi} \in \mathbb{N}^{L}} \gamma(\boldsymbol{k}, \boldsymbol{\xi}, x) e^{-i \boldsymbol{\omega} \cdot \boldsymbol{\xi}}\right]
$$

where $\gamma(\boldsymbol{k}, \boldsymbol{\xi}, x)$ then obeys

$$
\begin{aligned}
\gamma(\boldsymbol{k}, \boldsymbol{\xi}, x)= & \int d x^{\prime} p\left(x^{\prime}\right) Q\left(x, x^{\prime}\right) \sum_{\boldsymbol{k}^{\prime}} P\left(\boldsymbol{k}^{\prime} \mid x^{\prime}\right) \prod_{\ell=1}^{L} \delta_{\xi_{\ell}, k_{\ell-1}^{\prime}} \\
& \times \frac{\int_{-\pi}^{\pi} d \boldsymbol{\omega} \exp \left[i \sum_{\ell=1}^{L} \omega_{\ell}\left(k_{\ell}^{\prime}-k_{\ell-1}\right)+c \sum_{\boldsymbol{\xi}^{\prime} \in \mathbb{N}^{L}} \gamma\left(\boldsymbol{k}^{\prime}, \boldsymbol{\xi}^{\prime}, x^{\prime}\right) e^{\left.-i \boldsymbol{\omega} \cdot \boldsymbol{\xi}^{\prime}\right]}\right.}{\int_{-\pi}^{\pi} d \boldsymbol{\omega} \exp \left[i \boldsymbol{\omega} \cdot \boldsymbol{k}^{\prime}+c \sum_{\boldsymbol{\xi}^{\prime} \in \mathbb{N}^{L}} \gamma\left(\boldsymbol{k}^{\prime}, \boldsymbol{\xi}^{\prime}, x^{\prime}\right) e^{\left.-i \boldsymbol{\omega} \cdot \boldsymbol{\xi}^{\prime}\right]}\right.}
\end{aligned}
$$

The two integrals over $\boldsymbol{\omega}$ in the latter fraction can be done. Both are of the form

$$
\begin{aligned}
\mathcal{I}\left(\boldsymbol{k}, \boldsymbol{k}^{\prime}, x\right) & =\int_{-\pi}^{\pi} d \boldsymbol{\omega} \exp \left[i \boldsymbol{\omega} \cdot \boldsymbol{k}+c \sum_{\boldsymbol{\xi}^{\prime} \in \mathbb{N}^{L}} \gamma\left(\boldsymbol{k}^{\prime}, \boldsymbol{\xi}^{\prime}, x^{\prime}\right) e^{-i \boldsymbol{\omega} \cdot \boldsymbol{\xi}^{\prime}}\right] \\
& =(2 \pi)^{L} \sum_{m \geq 0} \frac{c^{m}}{m !} \sum_{\boldsymbol{\xi}^{1} \ldots \boldsymbol{\xi}^{m} \in \mathbb{N}^{L}}\left[\prod_{n=1}^{m} \gamma\left(\boldsymbol{k}^{\prime}, \boldsymbol{\xi}^{n}, x^{\prime}\right)\right] \delta \boldsymbol{k}_{, \sum_{n \leq m}} \boldsymbol{\xi}^{n}
\end{aligned}
$$

and hence the equation for $\gamma(\boldsymbol{k}, \boldsymbol{\xi}, x)$ becomes

$$
\begin{aligned}
& \gamma(\boldsymbol{k}, \boldsymbol{\xi}, x)=\delta_{\xi_{1}, 1} \int d x^{\prime} p\left(x^{\prime}\right) Q\left(x, x^{\prime}\right) \sum_{\boldsymbol{k}^{\prime}} P\left(\boldsymbol{k}^{\prime} \mid x^{\prime}\right) \prod_{\ell=1}^{L-1} \delta_{\xi_{\ell+1}, k_{\ell}^{\prime}} \\
& \times \frac{\sum_{m \geq 0} \frac{c^{m}}{m !} \sum_{\boldsymbol{\xi}^{1} \ldots \boldsymbol{\xi}^{m} \in \mathbb{N}^{L}}\left[\prod_{n=1}^{m} \gamma\left(\boldsymbol{k}^{\prime}, \boldsymbol{\xi}^{n}, x^{\prime}\right)\right] \prod_{\ell=1}^{L} \delta_{k_{\ell}^{\prime}, k_{\ell-1}+\sum_{n \leq m} \xi_{\ell}^{n}}}{\sum_{m \geq 0} \frac{c^{m}}{m !} \sum_{\boldsymbol{\xi}^{1} \ldots \boldsymbol{\xi}^{m} \in \mathbb{N}^{L}}\left[\prod_{n=1}^{m} \gamma\left(\boldsymbol{k}^{\prime}, \boldsymbol{\xi}^{n}, x^{\prime}\right)\right] \delta_{\boldsymbol{k}^{\prime}, \sum_{n \leq m}} \boldsymbol{\xi}^{n}} \\
& =\delta_{\xi_{1}, 1} \int d x^{\prime} p\left(x^{\prime}\right) Q\left(x, x^{\prime}\right) \sum_{\boldsymbol{k}^{\prime}} \frac{k_{1}^{\prime}}{c} P\left(\boldsymbol{k}^{\prime} \mid x^{\prime}\right) \prod_{\ell=1}^{L-1} \delta_{\xi_{\ell+1}, k_{\ell}^{\prime}} \\
& \times \frac{\sum_{\boldsymbol{\xi}^{1} \ldots \boldsymbol{\xi}^{k_{1}^{\prime}-1}}\left[\prod_{n=1}^{k_{1}^{\prime}-1} \gamma\left(\boldsymbol{k}^{\prime}, \boldsymbol{\xi}^{n}, x^{\prime}\right)\right] \prod_{\ell=1}^{L} \delta_{k_{\ell}^{\prime}, k_{\ell-1}+\sum_{n<k_{1}^{\prime}} \xi_{\ell}^{n}}}{\sum_{\boldsymbol{\xi}^{1} \ldots \boldsymbol{\xi}^{k_{1}^{\prime}}}\left[\prod_{n=1}^{k_{1}^{\prime}} \gamma\left(\boldsymbol{k}^{\prime}, \boldsymbol{\xi}^{n}, x^{\prime}\right)\right] \delta_{\boldsymbol{k}^{\prime}, \sum_{n \leq k_{1}^{\prime}} \boldsymbol{\xi}^{n}}}
\end{aligned}
$$

where we use the conventions that $\left[\prod_{n=1}^{m} u_{n}\right]_{m=0} \equiv 1,\left[\sum_{n=1}^{m} u_{n}\right]_{m=0} \equiv 0$, and $\left[\sum_{\xi_{1} \ldots \xi_{m}} u\left(\xi_{1}, \ldots, \xi_{m}\right)\right]_{m=0} \equiv 1$. If $L=1$ we have $\boldsymbol{k}=\rightarrow k$ and $\boldsymbol{\xi} \rightarrow 1$, so $\gamma(\boldsymbol{k}, \boldsymbol{\xi}, x) \rightarrow \gamma(k, x)$. This describes the situation where the degrees are not generalized, but measure as usual only 
the number of direct links per node. Here our equation for $\gamma(\ldots)$ simplifies drastically to

$$
L=1: \quad \gamma(k, x)=\int d x^{\prime} p\left(x^{\prime}\right) Q\left(x, x^{\prime}\right) \sum_{k^{\prime}} k^{\prime} \frac{P\left(k^{\prime} \mid x^{\prime}\right)}{c \gamma\left(k^{\prime}, x^{\prime}\right)}
$$

The right-hand side is clearly independent of $k$, so $\gamma(k, x)=\gamma(x)$ with

$$
\gamma(x)=\int d x^{\prime} p\left(x^{\prime}\right) \frac{Q\left(x, x^{\prime}\right)}{c \gamma\left(x^{\prime}\right)} \sum_{k} k P\left(k \mid x^{\prime}\right)
$$

If $L>1$ we can manipulate at most some further Kronecker $\delta$ s, and the final form is therefore

$$
\begin{aligned}
& \gamma(\boldsymbol{k}, \boldsymbol{\xi}, x)=\frac{\xi_{2} \delta_{\xi_{1}, 1}}{c} \int d x^{\prime} p\left(x^{\prime}\right) Q\left(x, x^{\prime}\right) \sum_{k^{\prime} \geq 0} P\left(\xi_{2}, \ldots, \xi_{L}, k^{\prime} \mid x^{\prime}\right) \\
& \times \frac{\sum_{\boldsymbol{\xi}^{1} \ldots \boldsymbol{\xi}^{\xi_{2}-1}}\left[\prod_{n=1}^{\xi_{2}-1} \gamma\left(\left(\xi_{2}, \ldots, \xi_{L}, k^{\prime}\right), \boldsymbol{\xi}^{n}, x^{\prime}\right)\right] \delta_{k^{\prime}, k_{L-1}+\sum_{n<\xi_{2}} \xi_{L}^{n}} \prod_{\ell=1}^{L-1} \delta_{\xi_{\ell+1}-k_{\ell-1}, \sum_{n<\xi_{2}} \xi_{\ell}^{n}}}{\sum_{\boldsymbol{\xi}^{1} \ldots \boldsymbol{\xi}^{\xi_{2}}}\left[\prod_{n=1}^{\xi_{2}} \gamma\left(\left(\xi_{2}, \ldots, \xi_{L}, k^{\prime}\right), \boldsymbol{\xi}^{n}, x^{\prime}\right)\right] \delta_{k^{\prime}, \sum_{n \leq \xi_{2}} \xi_{L}^{n}} \prod_{\ell=1}^{L-1} \delta_{\xi_{\ell+1}, \sum_{n \leq \xi_{2}}} \xi_{\ell}^{n}}
\end{aligned}
$$

\section{Simplification of the asymptotic entropy formulas}

At this stage we insert our previous results for the kernels $\{V, W, Q\}$ into (18], 19,20]21) to arrive at more explicit expressions for the asymptotic entropies, which will only involve the function $\gamma(\boldsymbol{k}, \boldsymbol{\xi}, x)$ of (36). The first step is to substitute expression (27) into (21). This leads, in combination with the fact that at the relevant saddle-points the kernels $\{V, W\}$ obey identical equations for the two cases (constrained generalized degrees versus constrained statistics of generalized degrees), to the simple and natural relation between our two entropies:

$$
\lim _{N \rightarrow \infty} \Omega_{L}[P \mid \boldsymbol{x}]=\lim _{N \rightarrow \infty} \Omega_{L}[\{\boldsymbol{k}\} \mid \boldsymbol{x}]-\int d x p(x) \sum_{\boldsymbol{k}} P(\boldsymbol{k} \mid x) \log P(\boldsymbol{k} \mid x)
$$

The extra freedom to construct microscopic network realizations in the case where we only constrain the generalized degree distribution, as opposed to constraining the actual values of the generalized degrees, is measured by the Shannon entropy of the imposed distribution $P(\boldsymbol{k} \mid x)$.

The relation (37) could be also derived from the definition of $\Omega[\ldots]$, given in (15)-(6). In fact we can observe that the probability $W(\boldsymbol{c} \mid \boldsymbol{x})$ present in the definition (55) of $\Omega[\{\boldsymbol{k}\} \mid \boldsymbol{x}]$ is invariant under all permutations of the labels of those nodes that have the same "hidden variable" $x$; this follows directly from definition (1). Consequently $\Omega_{L}[\{\boldsymbol{k}\} \mid \boldsymbol{x}]$ must also be invariant under any permutation of the labels of nodes with same value of $x$. It follows that 
$\Omega_{L}[\{\boldsymbol{k}\} \mid \boldsymbol{x}]$ is dependent on the degree sequence $\{\boldsymbol{k}\}$ only through the distributions $\{P(\boldsymbol{k} \mid x)\}$. Therefore, we can use this simple insight to predict the relation between $\Omega_{L}[\{\boldsymbol{k}\} \mid \boldsymbol{x}]$ and $\Omega_{L}[P \mid \boldsymbol{x}](37)$. In fact, because $\Omega_{L}[\{\boldsymbol{k}\} \mid \boldsymbol{x}]$ must be only dependent on the distribution $P(\boldsymbol{k} \mid x)$, we have that

$$
\begin{aligned}
\Omega_{L}[P \mid \boldsymbol{x}] & =\frac{1}{N} \log \sum_{\boldsymbol{k}_{1} \ldots \boldsymbol{k}_{N}} \prod_{\boldsymbol{k}, x} \delta\left[P(\boldsymbol{k} \mid x)-\frac{\sum_{i} \delta \boldsymbol{k}_{\boldsymbol{k}} \delta\left[x-x_{i}\right]}{N p(x)}\right] e^{N \Omega_{L}[\{\boldsymbol{k}\} \mid \boldsymbol{x}]} \\
& =\frac{1}{N} \log e^{N \Omega_{L}[\{\boldsymbol{k}\} \mid \boldsymbol{x}]} \prod_{x} \frac{[N p(x)] !}{\prod_{\mathbf{k}}[N p(x) P(\boldsymbol{k} \mid x)] !} .
\end{aligned}
$$

where $\{\boldsymbol{k}\}$ is any generalized degree sequence with degree distributions $P(\boldsymbol{k} \mid x)$. Using (38) we can derive relation (37).

In order to evaluate $\lim _{N \rightarrow \infty} \Omega_{L}[\{\boldsymbol{k}\} \mid \boldsymbol{x}]$ we only need to express $\lim _{N \rightarrow \infty} \Omega_{L}[\{\boldsymbol{k}\} \mid \boldsymbol{x}]$ in terms of the function $\gamma(\boldsymbol{k}, \boldsymbol{\xi}, x)$. We first note that at the relevant saddle-point the function $\Phi[\{W\}]$ (17) takes the value

$$
\Phi[\{W\}]=\frac{1}{2} \int d x \int_{-\pi}^{\pi} d \boldsymbol{\omega} \sum_{\boldsymbol{k}} W(\boldsymbol{\omega}, \boldsymbol{k}, x) \log V(\boldsymbol{\omega}, \boldsymbol{k}, x)
$$

Insertion into (20), followed by elimination of $W(\boldsymbol{\omega}, \boldsymbol{k}, x)$ via (23), leads us to

$$
\begin{aligned}
\lim _{N \rightarrow \infty} \Omega_{L}[\{\boldsymbol{k}\} \mid \boldsymbol{x}]= & \int d x p(x) \sum_{\boldsymbol{k}} P(\boldsymbol{k} \mid x) \log \int_{-\pi}^{\pi} \frac{d \boldsymbol{\omega}}{(2 \pi)^{L}} V(\boldsymbol{\omega}, \boldsymbol{k}, x) e^{i \boldsymbol{\omega} \cdot \boldsymbol{k}} \\
& -\frac{1}{2} \int d x p(x) \sum_{\boldsymbol{k}} P(\boldsymbol{k} \mid x) \frac{\int_{-\pi}^{\pi} d \boldsymbol{\omega} e^{i \boldsymbol{\omega} \cdot \boldsymbol{k}} V_{0}(\boldsymbol{\omega}, \boldsymbol{k}, x) \log V(\boldsymbol{\omega}, \boldsymbol{k}, x)}{\int_{-\pi}^{\pi} d \boldsymbol{\omega} V_{0}(\boldsymbol{\omega}, \boldsymbol{k}, x) e^{i \boldsymbol{\omega} \cdot \boldsymbol{k}}}
\end{aligned}
$$

where $V_{0}(\boldsymbol{\omega}, \boldsymbol{k}, x)=V(\boldsymbol{\omega}, \boldsymbol{k}, x) \exp \left[c \int d x^{\prime} Q\left(x, x^{\prime}\right) p\left(x^{\prime}\right)\right]$. The final step is the elimination of $V(\boldsymbol{\omega}, \boldsymbol{k}, x)$ via (29), followed by integration over $\boldsymbol{\omega}$, using the property that $\gamma(\boldsymbol{k}, \boldsymbol{\xi}, x)=0$ unless $\xi_{1}=1$ :

$$
\begin{gathered}
\int_{-\pi}^{\pi} \frac{d \boldsymbol{\omega}}{(2 \pi)^{L}} V_{0}(\boldsymbol{\omega}, \boldsymbol{k}, x) e^{i \boldsymbol{\omega} \cdot \boldsymbol{k}}=\sum_{m \geq 0} \frac{c^{m}}{m !} \sum_{\boldsymbol{\xi}^{1} \ldots \boldsymbol{\xi}^{m}} \prod_{n \leq m}\left[\gamma\left(\boldsymbol{k}, \boldsymbol{\xi}^{n}, x\right)\right] \delta_{\boldsymbol{k}, \sum_{n \leq m}} \boldsymbol{\xi}^{n} \\
=\delta_{\boldsymbol{k}, \boldsymbol{0}}+\frac{c^{k_{1}} \theta\left[k_{1}-\frac{1}{2}\right]}{k_{1} !} \sum_{\boldsymbol{\xi}^{1} \ldots \boldsymbol{\xi}^{k_{1}}} \prod_{n \leq k_{1}}\left[\gamma\left(\boldsymbol{k}, \boldsymbol{\xi}^{n}, x\right)\right] \delta_{\boldsymbol{k}, \sum_{n \leq k_{1}} \boldsymbol{\xi}^{r}(41)} \\
\int_{-\pi}^{\pi} \frac{d \boldsymbol{\omega}}{(2 \pi)^{L}} V_{0}(\boldsymbol{\omega}, \boldsymbol{k}, x) \log V_{0}(\boldsymbol{\omega}, \boldsymbol{k}, x) e^{i \boldsymbol{\omega} \cdot \boldsymbol{k}}=\sum_{m>0} \frac{c^{m}}{(m-1) !} \sum_{\boldsymbol{\xi}^{1} \ldots \boldsymbol{\xi}^{m}} \prod_{n \leq m}\left[\gamma\left(\boldsymbol{k}, \boldsymbol{\xi}^{n}, x\right)\right] \delta_{\boldsymbol{k}, \sum_{n \leq m}} \boldsymbol{\xi}^{n} \\
=\frac{c^{k_{1}} \theta\left[k_{1}-\frac{1}{2}\right]}{\left(k_{1}-1\right) !} \sum_{\boldsymbol{\xi}^{1} \ldots \boldsymbol{\xi}^{k_{1}}} \prod_{n \leq k_{1}}\left[\gamma\left(\boldsymbol{k}, \boldsymbol{\xi}^{n}, x\right)\right] \delta_{\boldsymbol{k}, \sum_{n \leq k_{1}} \boldsymbol{\xi}^{n}}
\end{gathered}
$$


So one arrives at the compact result, where we have used the fact that if $k_{1}=0$ then $k_{\ell}=0$ for all $\ell$ (which follows from the definition of the generalized degrees):

$$
\begin{array}{r}
\lim _{N \rightarrow \infty} \Omega_{L}[\{\boldsymbol{k}\} \mid \boldsymbol{x}]=\sum_{k_{1}} P\left(k_{1}\right) \log \pi_{c}\left(k_{1}\right)+\frac{1}{2}\left[c-\int d x p(x) \sum_{\boldsymbol{k}} k_{1} P(\boldsymbol{k} \mid x)\right] \\
+\int d x p(x) \sum_{\boldsymbol{k}} P(\boldsymbol{k} \mid x) \log \left\{\sum_{\boldsymbol{\xi}^{1} \ldots \boldsymbol{\xi}^{k_{1}}}\left[\prod_{n \leq k_{1}} \gamma\left(\boldsymbol{k}, \boldsymbol{\xi}^{n}, x\right)\right] \delta_{\boldsymbol{k}, \sum_{n \leq k_{1}} \boldsymbol{\xi}^{n}}\right\}
\end{array}
$$

with the average-c Poissonian degree distribution $\pi_{c}(k)=c^{k} e^{-c} / k !$.

\section{APPLICATIONS OF THE GENERAL THEORY}

\section{A. Regular random graphs}

Our first application domain is that of $r$-regular degree distribution $P(\boldsymbol{k} \mid x)=\delta_{\boldsymbol{k}, \boldsymbol{k}(r)}$, with $\boldsymbol{k}(r)=\left(r, r^{2}, \ldots, r^{L}\right)$. Here one can solve (36) explicitly:

$$
\begin{aligned}
& \gamma(\boldsymbol{k}, \boldsymbol{\xi}, x)=\frac{\xi_{2} \delta_{\xi_{1}, 1}}{c} \int d x^{\prime} p\left(x^{\prime}\right) Q\left(x, x^{\prime}\right) \sum_{k^{\prime} \geq 0} \delta_{\left(\xi_{2}, \ldots, \xi_{L}, k^{\prime}\right), \boldsymbol{k}(r)}
\end{aligned}
$$

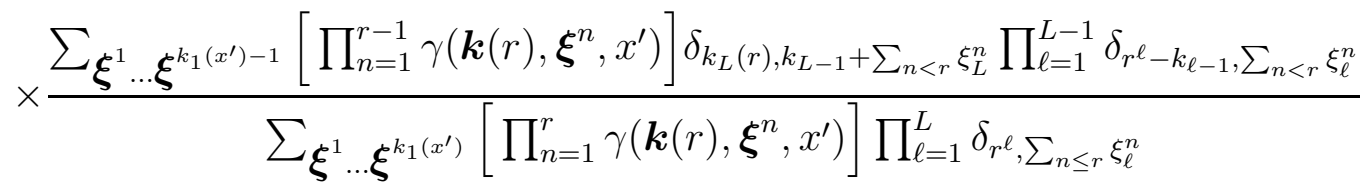

The solution is seen to be of the form $\gamma(\boldsymbol{k}, \boldsymbol{\xi}, x)=\gamma(\boldsymbol{k}, x) \delta_{\boldsymbol{\xi},\left(1, r, r^{2}, \ldots, r^{L-1}\right)}$, and independent of $k_{L}$. Insertion of this form into the above equation then gives

$$
\gamma(\boldsymbol{k}, x)=\frac{r}{c} \int d x^{\prime} p\left(x^{\prime}\right) Q\left(x, x^{\prime}\right) \frac{\prod_{\ell=1}^{L-1} \delta_{k_{\ell}, r}}{\gamma\left(\boldsymbol{k}(r), x^{\prime}\right)}
$$

We conclude that

$$
\gamma(\boldsymbol{k}, \boldsymbol{\xi}, x)=\gamma(x)\left[\prod_{\ell=1}^{L-1} \delta_{k_{\ell}, r^{\ell}}\right]\left[\prod_{\ell=1}^{L} \delta_{\xi_{\ell}, r^{\ell-1}}\right]
$$

where $\gamma(x)$ is the solution of

$$
\gamma(x)=\frac{r}{c} \int d x^{\prime} p\left(x^{\prime}\right) \frac{Q\left(x, x^{\prime}\right)}{\gamma\left(x^{\prime}\right)}
$$

For the entropies (37, 133 ) one then finds

$$
\lim _{N \rightarrow \infty} \Omega_{L}[P \mid \boldsymbol{x}]=\lim _{N \rightarrow \infty} \Omega_{L}[\{\boldsymbol{k}\} \mid \boldsymbol{x}]=\log \pi_{c}(r)+r \int d x p(x) \log \gamma(x)+\frac{1}{2}(c-r)
$$


As expected, the two entropies are identical (since for regular graphs there is no entropy contribution from degree permutations) and independent of $L$ (since upon specifying that the degrees are $r$-regular, the full distributions $P(\boldsymbol{k} \mid x)$ are uniquely specified for any $L)$. In the special case $Q\left(x, x^{\prime}\right)=1$ of uncorrelated degrees the above solution simplifies further. Now $\gamma(x)=\sqrt{r / c}$, and

$$
\lim _{N \rightarrow \infty} \Omega_{L}[P|\boldsymbol{x}| \boldsymbol{x}]=\lim _{N \rightarrow \infty} \Omega_{L}[\{\boldsymbol{k}\} \mid \boldsymbol{x}]=\log \pi_{c}(r)+\frac{1}{2} r \log (r / c)+\frac{1}{2}(c-r)
$$

\section{B. The case $L=1$}

For $L=1$ we have already simplified our formula for the function $\gamma(\boldsymbol{k}, \boldsymbol{\xi}, x)$ to relation (35) for a simple function $\gamma(x)$. We can do the same for expression (43) for the entropy, which gives

$$
\begin{aligned}
\lim _{N \rightarrow \infty} \Omega_{1}[\{k\} \mid \boldsymbol{x}] & =\sum_{k} P(k) \log \pi_{c}(k)+\frac{1}{2}(c-\bar{k})+\int d x p(x) \sum_{k} P(k \mid x) \log \gamma^{k}(x) \\
\lim _{N \rightarrow \infty} \Omega_{1}[P \mid \boldsymbol{x}] & =\sum_{k} P(k) \log \pi_{c}(k)+\frac{1}{2}(c-\bar{k})-\int d x p(x) \sum_{k} P(k \mid x) \log \left[P(k \mid x) / \gamma^{k}(x)\right]
\end{aligned}
$$

with $\pi_{c}(k)=c^{k} e^{-c} / k$ !, with $\bar{k}=\int d x p(x) \sum_{k} k P(k \mid x), P(k)=\int d x p(x) P(k \mid x)$ and where $\gamma(x)$ is to be solved from

$$
\gamma(x)=\int d x^{\prime} p\left(x^{\prime}\right) \frac{Q\left(x, x^{\prime}\right)}{c \gamma\left(x^{\prime}\right)} \sum_{k} k P\left(k \mid x^{\prime}\right)
$$

We see immediately that for $Q\left(x, x^{\prime}\right)=1$ (the Erdös-Rényi ensemble), and upon choosing $P(k \mid x)=P(k)$ (since for $Q\left(x, x^{\prime}\right)=1$ the hidden variables $x$ are obsolete) we would have had $\gamma(x)=\sqrt{\bar{k} / c} \forall x$. Expression (501) now becomes

$$
\begin{array}{ll}
\lim _{N \rightarrow \infty} \Omega_{1}[\{k\} \mid \boldsymbol{x}]=\sum_{k} P(k) \log \pi_{c}(k)+\frac{1}{2}(c-\bar{k})+\frac{1}{2} \bar{k} \log (\bar{k} / c) \\
\lim _{N \rightarrow \infty} \Omega_{1}[P \mid \boldsymbol{x}]=-\sum_{k} P(k) \log \left[P(k) / \pi_{c}(k)\right]+\frac{1}{2}(c-\bar{k})+\frac{1}{2} \bar{k} \log (\bar{k} / c)
\end{array}
$$

So, if one also chooses $\bar{k}=c$, the entropy of networks with degree distribution $P(k)$ in the Erdös-Rényi ensemble is minus the Kullback-Leibler distance between $P(k)$ and a Poisson degree distribution, provided the ensemble and $P(k)$ have the same average connectivity. An alternative derivation of equation (53) can also be obtained starting from the expression of the total number of graphs with given degree sequence $\mathcal{N}[\{\boldsymbol{k}\}]$ derived in $[10,13,15]$ :

$$
\mathcal{N}[\{\boldsymbol{k}\}]=(\bar{k} N-1) ! ! \frac{e^{-\frac{1}{4} \lambda}}{\prod_{i} k_{i} !}
$$


with $\lambda=\left(\overline{k^{2}} / \bar{k}\right)^{2}-1$ and $\overline{k^{2}}=\sum_{i} k_{i}^{2} / N$. The entropy $\Omega[\{\boldsymbol{k}\}]$ of the degree sequence $\{\boldsymbol{k}\}$ in the Erdös-Rényi ensemble, is the logarithm of the probability of having one of the total number $\mathcal{N}[\{k\}]$ of possible networks in the ensemble. Since in a Erdös-Rényi network each link has a probability $c / N$ to be present, we have

$$
\lim _{N \rightarrow \infty} \Omega_{1}[\{\boldsymbol{k}\}]=\lim _{N \rightarrow \infty} \frac{1}{N} \log \left\{\mathcal{N}[\{k\}]\left(\frac{c}{N}\right)^{N \bar{k} / 2}\left(1-\frac{c}{N}\right)^{N(N-1) / 2-N \bar{k} / 2}\right\} .
$$

Upon inserting the expression of $\mathcal{N}[\{\boldsymbol{k}\}]$, (54) in Eq. (55) we recover (53).

The other terms in (50) apparently represent the effect of average connectivity mismatches and of the degree correlations induced by $Q(.,$.$) , and make matters more complicated. The$ simple form of our $L=1$ equations, however, still allows us to push the analysis further for certain cases, by solving $\gamma(x)$ explicitly from equation (52). For instance, if the (symmetric) kernel $Q\left(x, x^{\prime}\right)$ has an eigenfunction $f(x)=\sqrt{p(x) k(x)}$, with $k(x)=\sum_{k} k P(k \mid x)$ then

$$
\int d x^{\prime} Q\left(x, x^{\prime}\right) f\left(x^{\prime}\right)=\lambda f(x), \quad f(x)=\sqrt{p(x) k(x)}: \quad \gamma(x)=\sqrt{\frac{\lambda}{c}} f(x)
$$

If $Q(.,$.$) has this property, together with the normalization \int d x d x^{\prime} p(x) Q\left(x, x^{\prime}\right) p\left(x^{\prime}\right)=1$, then one finds that the entropy (50) becomes

$$
\begin{aligned}
\left.\lim _{N \rightarrow \infty} \Omega_{1}[\{k\}] \mid \boldsymbol{x}\right] & =\sum_{k} P(k) \log \pi_{c}(k)+\frac{1}{2}(c-\bar{k})+\frac{1}{2} \int d x p(x) k(x) \log [\lambda p(x) k(x) / c] \\
& =\sum_{k} P(k) \log \pi_{c}(k)+\frac{1}{2}(c-\bar{k})+\frac{1}{2} \int d x p(x) k(x) \log [p(x) k(x)]+\frac{1}{2} \bar{k} \log [\lambda / c]
\end{aligned}
$$

(where $\bar{k}=\int d x p(x) k(x)$ ). Let us next discuss some example kernels $Q\left(x, x^{\prime}\right)$ for which $\gamma(x)$ can be solved explicitly, either directly, or via the above procedure based on using eigenfunctions of $Q(.,$.$) :$

- First example:

Here we assume $Q\left(x, x^{\prime}\right)$ to be such that the conditional connectivities $k(x)=$ $\sum_{k} k P(k \mid x)$ are the typical ones for the ensemble (1), which implies that

$$
k(x)=c \int d x^{\prime} Q\left(x, x^{\prime}\right) p\left(x^{\prime}\right)
$$

and $\bar{k}=c$. In this case (52) has the solution $\gamma(x)=k(x) / c$, which leads to the following simple expression for the entropies:

$$
\lim _{N \rightarrow \infty} \Omega_{1}[\{k\} \mid \boldsymbol{x}]=\int d x p(x) \sum_{k} P(k \mid x) \log \pi_{k(x)}
$$




$$
\lim _{N \rightarrow \infty} \Omega_{1}[P \mid \boldsymbol{x}]=-\int d x p(x) \sum_{k} P(k \mid x) \log \left[P(k \mid x) / \pi_{k(x)}\right]
$$

This indicates that in this case the entropy $\lim _{N \rightarrow \infty} \Omega_{1}[P \mid \boldsymbol{x}]$ takes the form of an integral over $p(x)$ of the Kullback-Leibler distance between the probabilities $P(k \mid x)$ and the Possion distribution $\pi_{k(x)}$. We note that for the hidden variable model the typical degree distribution of the nodes with hidden variable $x$ is indeed $\pi_{k(x)}$ [20].

- Second example:

$$
Q\left(x, x^{\prime}\right)=a_{0}+a_{1} \delta\left(x-x^{\prime}\right), \quad k(x)=\frac{1}{2} c / p(x)
$$

with $x \in[-1,1]$. Normalization of $Q(.,$.$) tells us that a_{0}=1-a_{1} \int d x p^{2}(x)$, and we need $0 \leq a_{1} \leq\left[\int d x p^{2}(x)\right]^{-1}$ to ensure non-negative bond probabilities in our network ensemble. The networks in this ensemble have a non trivial community structure. In fact nodes with same hidden-variable have a larger probability to be connected. Here one finds a solution with $\bar{k}=c$ and $\gamma(x)=\gamma$, where

$$
\begin{aligned}
& \gamma=\sqrt{1-a_{1} \int d x p^{2}(x)+\frac{1}{2} a_{1}} \\
& \lim _{N \rightarrow \infty} \Omega_{1}[\{k\} \mid \boldsymbol{x}]=\sum_{k} P(k) \log \pi_{c}(k)+\frac{1}{2} c \log \left[1-a_{1} \int_{-1}^{1} d x p^{2}(x)+\frac{1}{2} a_{1}\right]
\end{aligned}
$$

- Third example:

$$
Q\left(x, x^{\prime}\right)=\frac{g(x)+g\left(x^{\prime}\right)}{2 \int d x^{\prime \prime} p\left(x^{\prime \prime}\right) g\left(x^{\prime \prime}\right)}, \quad k(x)=\frac{\left[\sqrt{\left\langle g^{2}\right\rangle_{0}}+g(x)\right]^{2}}{p(x)}
$$

with $x \in[-1,1]$, with the short-hand $\langle\phi\rangle_{0}=\frac{1}{2} \int_{-1}^{1} d x \phi(x)$, and with $g(x) \geq 0$ for all $x \in[-1,1]$. Here one finds the solution

$$
\gamma(x)=\frac{1}{\sqrt{c}} \sqrt{\frac{\langle g\rangle_{0}+\sqrt{\left\langle g^{2}\right\rangle_{0}}}{\int d x^{\prime} p\left(x^{\prime}\right) g\left(x^{\prime}\right)}}\left[\sqrt{\left\langle g^{2}\right\rangle_{0}}+g(x)\right], \quad \lambda=\frac{\langle g\rangle_{0}+\sqrt{\left\langle g^{2}\right\rangle_{0}}}{\int d x p(x) g(x)}
$$

\section{The case $L=2$}

Here we have to find first the solution of (36), which now reduces to

$$
\begin{aligned}
\gamma\left(\left(k_{1}, k_{2}\right),(1, \xi), x\right)= & \frac{\xi}{c} \int d x^{\prime} p\left(x^{\prime}\right) Q\left(x, x^{\prime}\right) \sum_{k^{\prime} \geq 0} P\left(\xi, k^{\prime} \mid x^{\prime}\right) \\
& \times \frac{\sum_{\xi_{1} \ldots \xi_{\xi-1}}\left[\prod_{n=1}^{\xi-1} \gamma\left(\left(\xi, k^{\prime}\right),\left(1, \xi_{n}\right), x^{\prime}\right)\right] \delta_{k^{\prime}, k_{1}+\sum_{n<\xi} \xi_{n}}}{\sum_{\xi_{1} \ldots \xi_{\xi}}\left[\prod_{n=1}^{\xi} \gamma\left(\left(\xi, k^{\prime}\right),\left(1, \xi_{n}\right), x^{\prime}\right)\right] \delta_{k^{\prime}, \sum_{n \leq \xi} \xi_{n}}}
\end{aligned}
$$


We observe that the right-hand side is independent of $k_{2}$, so the solution of our equation must have the form $\gamma\left(\left(k_{1}, k_{2}\right),(1, \xi), x\right)=\gamma\left(k_{1}, \xi, x\right)$, where

$$
\begin{aligned}
\gamma(k, \xi, x)= & \frac{\xi}{c} \int d x^{\prime} p\left(x^{\prime}\right) Q\left(x, x^{\prime}\right) \sum_{k^{\prime} \geq 0} P\left(\xi, k^{\prime} \mid x^{\prime}\right) \\
& \times \frac{\sum_{\xi_{1} \ldots \xi_{\xi-1}}\left[\prod_{n=1}^{\xi-1} \gamma\left(\xi, \xi_{n}, x^{\prime}\right)\right] \delta_{k^{\prime}, k+\sum_{n<\xi} \xi_{n}}}{\sum_{\xi_{1} \ldots \xi_{\xi}}\left[\prod_{n=1}^{\xi} \gamma\left(\xi, \xi_{n}, x^{\prime}\right)\right] \delta_{k^{\prime}, \sum_{n \leq \xi} \xi_{n}}} .
\end{aligned}
$$

The entropy would become

$$
\begin{aligned}
\lim _{N \rightarrow \infty} \Omega_{2}[\{\boldsymbol{k}\} \mid \boldsymbol{x}] & =\sum_{k_{1}} P\left(k_{1}\right) \log \pi_{c}\left(k_{1}\right)+\frac{1}{2}\left[c-\int d x p(x) \sum_{k_{1} k_{2}} k_{1} P\left(k_{1}, k_{2} \mid x\right)\right] \\
& +\int d x p(x) \sum_{k_{1} k_{2}} P\left(k_{1}, k_{2} \mid x\right) \log \left\{\sum_{\xi_{1} \ldots \xi_{k_{1}}}\left[\prod_{n \leq k_{1}} \gamma\left(k_{1}, \xi_{n}, x\right)\right] \delta_{k_{2}, \sum_{n \leq k_{1}} \xi_{n}}\right\}
\end{aligned}
$$

Let us limit ourselves to the simplest scenario where there are no degree correlations, i.e. $Q\left(x, x^{\prime}\right)=1$. Here we have $\gamma(k, \xi, x)=\gamma(k, \xi)$, and we need only the generalized degree statistics $P\left(k_{1}, k_{2}\right)=\int d x p(x) P\left(k_{1}, k_{2} \mid x\right)$. Our formulae thereby reduce to

$$
\begin{aligned}
\gamma(k, \xi) & =\frac{\xi}{c} \sum_{k^{\prime} \geq 0} P\left(\xi, k^{\prime}\right) \frac{\sum_{\xi_{1} \ldots \xi_{\xi-1}}\left[\prod_{n=1}^{\xi-1} \gamma\left(\xi, \xi_{n}\right)\right] \delta_{k^{\prime}, k+\sum_{n<\xi} \xi_{n}}}{\sum_{\xi_{1} \ldots \xi_{\xi}}\left[\prod_{n=1}^{\xi} \gamma\left(\xi, \xi_{n}\right)\right] \delta_{k^{\prime}, \sum_{n \leq \xi} \xi_{n}}} \\
\lim _{N \rightarrow \infty} \Omega_{2}[\{\boldsymbol{k}\} \mid \boldsymbol{x}] & =\sum_{k_{1}} P\left(k_{1}\right) \log \pi_{c}\left(k_{1}\right)+\frac{1}{2}\left[c-\sum_{k_{1} k_{2}} k_{1} P\left(k_{1}, k_{2}\right)\right] \\
+ & \sum_{k_{1} k_{2}} P\left(k_{1}, k_{2}\right) \log \left\{\sum_{\xi_{1} \ldots \xi_{k_{1}}}\left[\prod_{n \leq k_{1}} \gamma\left(k_{1}, \xi_{n}\right)\right] \delta_{k_{2}, \sum_{n \leq k_{1}} \xi_{n}}\right\}
\end{aligned}
$$

Here one observes the validity of the following simple relation:

$$
\sum_{k_{2}} \gamma\left(k_{1}, k_{2}\right) \gamma\left(k_{2}, k_{1}\right)=\frac{k_{1}}{c} P\left(k_{1}\right)
$$

\section{CONCLUSIONS}

In conclusion, we have calculated the entropies $\Omega_{L}[\{\boldsymbol{k}\} \mid \boldsymbol{x}]$ and $\Omega_{L}[P \mid \boldsymbol{x}]$ of hierarchical constrained network topologies in the "canonical" ensemble of large sparse networks described in terms of "hidden variables".

The expression of the entropy $\Omega_{L}[P \mid \boldsymbol{x}]$ assumes a very clear form in the case in which the network topology under study is the degree distribution of a network of the ensemble. 
Here the entropy measures the large deviation of the topology of the given networks from the typical topology of networks in the chosen ensemble.

The entropy measures the likelihood that a particular network topology belongs to an ensemble, as such it is an important quantity whenever one seeks to represent or characterize observed networks in terms of appropriate random network ensembles. We therefore believe that it may have many applications in the future in the context of community detection problems as well as other inference problems on complex networks.

\section{Acknowledgements}

We thank our anonymous referees for suggesting useful alternative derivations in places, and for noting an error in the first version. One of the authors (CJPV) acknowledges financial support from project FIS2006-13321-C02-01) and grant PR2006-0458. This work was also supported by the project IST STREP GENNETEC contract No.034952.

[1] A. C. C. Coolen, R. Kühn and P. Sollich Theory of Neural Information Processing Systems, (Oxford University Press, Oxford,2005).

[2] C. Beck and F. Shlög, Thermodynamics of chaotic systems (Cambridge University Press, Cambridge,1993).

[3] M. Tummiello, F. Lillo and R. N. Mantegna, Phys. Rev. E 76, 031123 (2007).

[4] G. Biroli, J.-P. Bouchaud and M. Potters, arXiv:0710.0802[cond-mat] (2007).

[5] A. Engel, R. Monasson and A. K. Hartmann, Jour. Stat. Phys. 117, 387 (2004).

[6] O. Rivoire, Jour. Stat. Phys. 117, 453 (2004).

[7] R. Albert and A.-L. Barabási, Rev. Mod. Phys. 74, 47 (2002).

[8] S. N. Dorogovtsev, A. Goltsev and J. F. F. Mendes, arXiv:0705.0010 [cond-mat] (2007).

[9] S. Boccaletti, V. Latora, Y. Moreno, M. Chavez and D. U. Hwang, Phys. Rep. 424, 175 (2006).

[10] G. Bianconi, Europhys. Lett. 81, 28005 (2008).

[11] J. Gomez-Gardenes and V. Latora, arXiv:0712.0278[cond-mat].

[12] M. E. J. Newman and E. A. Leicht, PNAS 104, 9564 (2007). 
[13] E. Bender and E. Rodney Canfield, J. Combin. Theory Ser. A 24, 296 (1978).

[14] B. D. McKay, Ars Combin. 19A, 15 (1985).

[15] B. Pittel and N. Wormald, J. Combin. Theory Ser. A 101, 249 (2003).

[16] J. Park and M. E. J. Newman, Phys. Rev. E 70, 066146 (2004).

[17] B. Sodeberg, Phys. Rev. E 66, 066121 (2002).

[18] F. Chung and L. Lu, PNAS 99, 15879 (2002).

[19] G. Caldarelli, A. Capocci, P. De Los Rios and M. A. Muñoz, Phys. Rev. Lett. 89, 258702 (2002).

[20] M. Boguñá and R. Pastor-Satorras, Phys. Rev. E 68, 036112 (2003).

[21] R. Monasson, J. Phys. A 31, 513 (1998).

[22] R. Monasson and R. Zecchina, Phys. Rev. E 56, 1357 (1997).

[23] R. Monasson and R. Zecchina, J. Phys. A: Math. Gen. 31, 9209 (1998).

[24] R. Monasson, R. Zecchina, S. Kirkpatrick, B. Selman and L. Troyansky Nature 400, 133 (1999).

[25] M. Weigt and A. K. Hartmann, Phys. Rev. Lett. 84, 6118 (2000).

[26] R. Mulet, A. Pagnani, M. Weigt and R. Zecchina, Phys. Rev. Lett. 89, 268701 (2002).

[27] M. Leone, A. Vàzquez, A. Vespignani and R. Zecchina, Eur. Phys. Jour. B 28, 191 (2002).

[28] B. Wemmenhove and A. C. C. Coolen, J. Phys. A: Math. Gen. 36, 9617 (2003).

[29] I. Pérez-Castillo and N. S. Skantzos, J. Phys. A: Math. Gen. 37, 9087 (2004).

[30] I. Pérez-Castillo, B. Wemmenhove, J. P. L. Hatchett, A. C. C. Coolen, N. S. Skantzos and T. Nikoletopoulos, J. Phys. A: Math. Gen. 37, 8789 (2004).

[31] T. Nikoletopoulos T, A. C. C. Coolen, I. Pérez Castillo,N. S. Skantzos, J. P. L. Hatchett and B. Wemmenhove J. Phys. A: Math. Gen. 37, 6455 (2004).

[32] T. Nikoletopoulos and A. C. C. Coolen, J. Phys. A: Math. Gen. 37, 8433 (2004).

[33] J. P. L. Hatchett, I. Pérez-Castillo, A. C. C. Coolen and N. S. Skantzos, Phys. Rev. Lett. 95, $117204(2005)$.

[34] H. Hansen-Goos and M. Weigt, J. Stat. Mech. Theory Exp. P08001 (2005).

[35] A. C. C. Coolen, N. S. Skantzos, I. Pérez Castillo, C. J. Pérez Vicente, J. P. L. Hatchett, B. Wemmenhove and T. Nikoletopoulos, J. Phys. A: Math. Gen. 38, 8289 (2005).

[36] N. S. Skantzos, I. Pérez Castillo and J. P. L. Hatchett, Phys. Rev. E 72, 066127 (2005).

[37] N. S. Skantzos and J. P. L. Hatchett, Physica A 381, 202 (2007). 
[38] A. Mozeika and A. C. C. Coolen, preprint arXiv:0712.1139 (2007).

[39] L. Zdeborova and F. Krzakala, Phys. Rev. E 76, 031131 (2007).

[40] J. Raymond and D. Saad, J. Phys. A: Math. Gen. 40, 12315 (2007).

[41] R. C. Alamino and D. Saad, J. Phys. A: Math. Gen. 40, 12259 (2007). 